
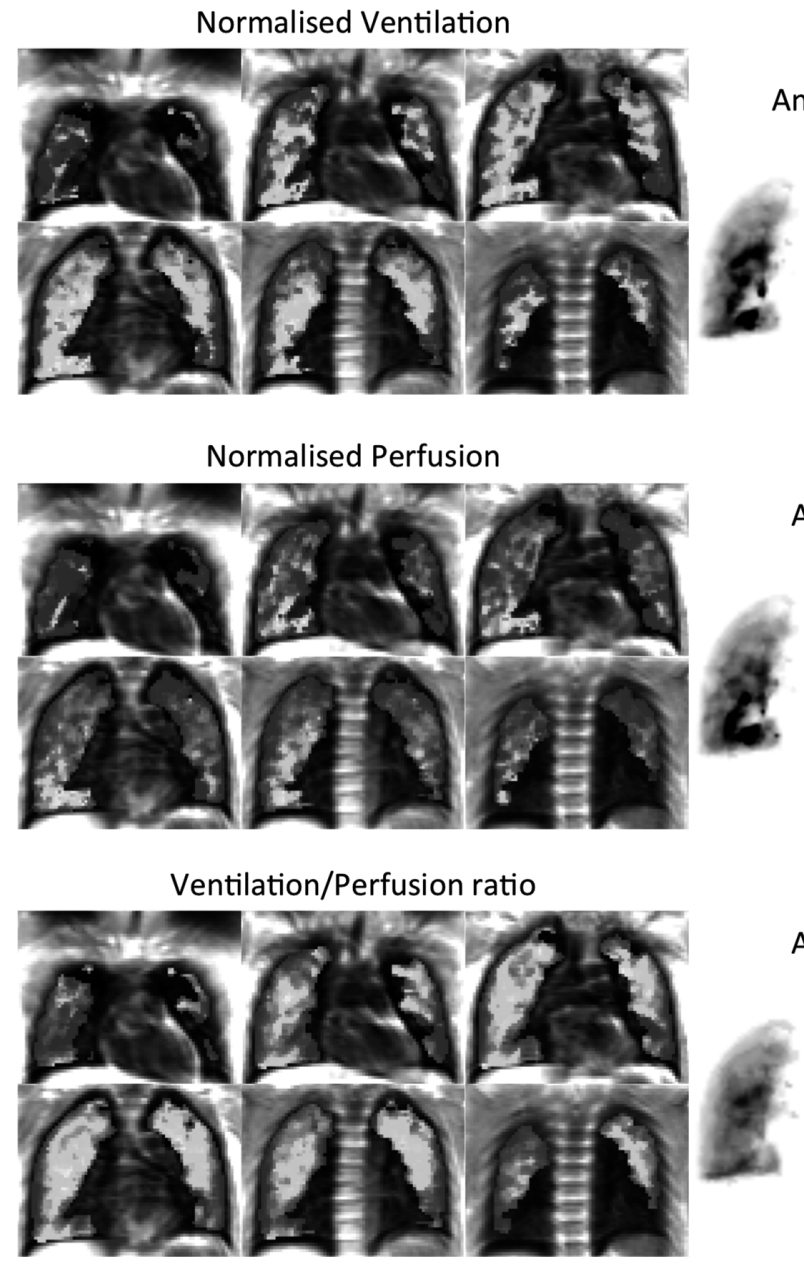

Abstract P284 Figure 1

\section{P285 HELIUM MAGNETIC RESONANCE IMAGING IDENTIFIES REGIONAL VENTILATION, PERFUSION AND MICROSTRUCTURE ABNORMALITIES IN A CASE OF "HORSE-SHOE" LUNG}

${ }^{1} \mathrm{~S}$ Zaidi, ${ }^{2} \mathrm{JM}$ Wild, ${ }^{2} \mathrm{~A}$ Swift, ${ }^{2} \mathrm{H}$ Marshall, ${ }^{2} \mathrm{~N}$ Weateherley, ${ }^{2} \mathrm{FC}$ Horn, ${ }^{1} \mathrm{RM}$ Niven, ${ }^{1} \mathrm{D}$ Ryan. ${ }^{1}$ University of Manchester, National Institute of Health Research, Respiratory and Allergy Clinical Research Facility, University Hospital South Manchester, Manchester, UK; ${ }^{2}$ Academic Radiology, University of Sheffield, Sheffield, UK

\subsection{6/thoraxjnl-2015-207770.421}

Introduction Helium magnetic resonance imaging $\left({ }^{3} \mathrm{He} \mathrm{MRI}\right)$ provides functional information on lung ventilation, perfusion and microstructure. We describe use of ${ }^{3} \mathrm{He}$ MRI to assess lung function in a patient with "horse-shoe" lung.

Case presentation EB is a 46 year old female with congenital lung abnormalities diagnosed during infancy. Chest $\mathrm{x}$-ray confirmed an abnormal right upper lobe (RUL). Pulmonary arteriography reported hypoplastic RUL vessels. Bronchoscopy confirmed tracheal stenosis, and cardiac catheterisation reported an abnormal pulmonary arterial pattern. A diagnosis of congenital lung anomalies along with asthma was made during her childhood. Throughout her life her condition has been characterised by recurrent severe respiratory tract infections requiring hospitalisation.

Recent CT chest confirmed right lung hypoplasia a deformed right main bronchus, moderate hypoplasia of the right main pulmonary artery and severe hypoplasia of RUL pulmonary vessels.
Projections of Ventilation map

Posterior Right-Anterior- Left-AnteriorOblique (RAO) Oblique (LAO)
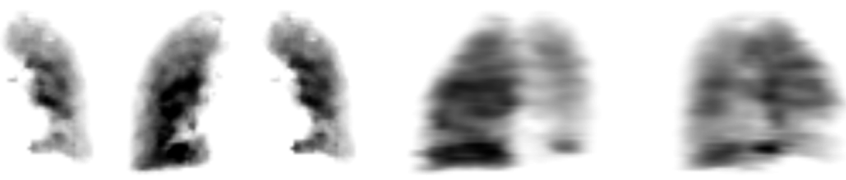

Projections of Perfusion map

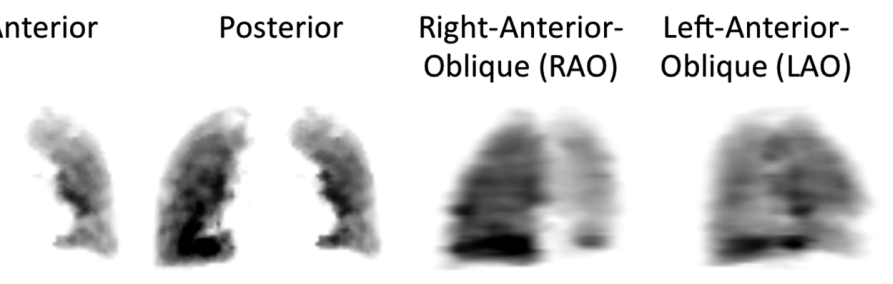

Projection of ventilation/perfusion map

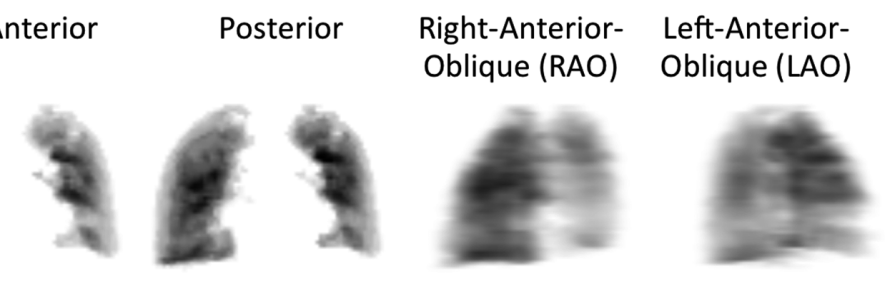

An isthmus joins the two lungs consistent with "horse-shoe lung". ${ }^{1}$ Ventilation-perfusion scan reported 26\% functioning right lung. Bronchoscopy confirmed tracheal and right main stem stenosis with left main stem bronchomalacia.

Surgical resection is an option for 'horse-shoe 'lung characterised by recurrent infections. ${ }^{2}$ In this context, we proceeded to ${ }^{3} \mathrm{He}$ MRI to obtain more detailed functional information.
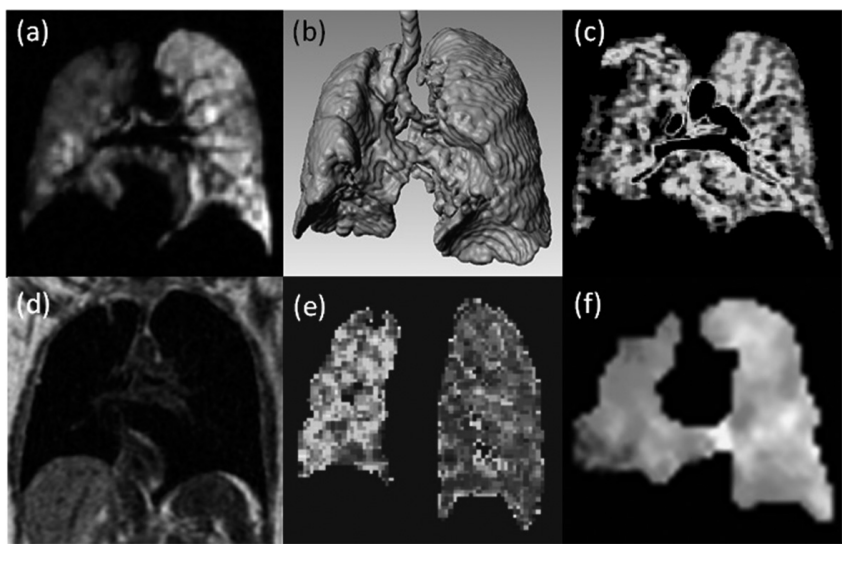

Abstract P285 Figure 1 Results of 3He functional MRI

Results of ${ }^{3} \mathrm{He}$ Functional MRI Communication between the right and left lungs was visualised (Figure 1). In the right lung, ventilation was reduced with increased heterogeneity (Figure 1a, 
f). And perfusion notably reduced, in the RUL (Figure 1c. ${ }^{3} \mathrm{He}$ apparent diffusion coefficient (ADC), which estimates acinar airway dimensions, was elevated and heterogeneous (Figure 1c), consistent with hypoplasia. The left lung was well ventilated and perfused with normal ADC values.

Conclusion ${ }^{3} \mathrm{He}$ MRI shows promise in functional analysis of "horse-shoe" lung with hypoplasia. This can prove useful in surgical assessment of these patients and therefore improve their management.

\section{REFERENCES}

1 Dikensoy O, Kervancioglu R, Bayram NG, Elbek O, Uyar M, Ekinci E. Horseshoe lung associated with scimitar syndrome and pleural lipoma. I Thorac Imaging 2006;21(1):73-5

2 Orzan F, Angelini P, Oglietti J, Leachman RD, Cooley DA. Horses lung: report of two cases. Am Heart J 1977;93(4):501-5

\section{P286 CORRELATIONS OF FUNCTIONAL MULTI-NUCLEAR MR IMAGING INDICES WITH PULMONARY FUNCTION TESTS IN THE ASSESSMENT OF IDIOPATHIC PULMONARY FIBROSIS}

${ }^{1} \mathrm{ND}$ Weatherley, ${ }^{1} \mathrm{NJ}$ Stewart, ${ }^{1} \mathrm{H}$ Marshall, ${ }^{1} \mathrm{G}$ Collier, ${ }^{1} \mathrm{~K}$ Hart, ${ }^{1} \mathrm{~F}$ Horn, ${ }^{1} \mathrm{G}$ Norquay, ${ }^{1}$ MK Whyte, ${ }^{2} S$ Bianchi, 'JM Wild. 'University of Sheffield, Sheffield, UK; ${ }^{2}$ Sheffield Teaching Hospitals, Sheffield, UK

\subsection{6/thoraxjnl-2015-207770.422}

Disease progression in idiopathic pulmonary fibrosis (IPF) is variable and unpredictable. Declining forced vital capacity (FVC) and transfer factor (TLCO) of $10 \%$ and $15 \%$ respectively are common markers of deterioration, but may be insufficiently sensitive to prognosticate and rely on serial measurements. Echocardiography provides screening for pulmonary artery hypertension (PAH), but is insensitive to pulmonary haemodynamic change before $\mathrm{PAH}$ is already apparent. Recently established Magnetic Resonance Imaging (MRI) techniques may provide insight into evaluating IPF. Greater septal thickness as measured by hyperpolarised xenon in patients with IPF compared with with healthy volunteers was previously demonstrated. ${ }^{1}$ Here, we investigate the correlation between MRI indices, including hyperpolarised gas ventilation and gadolinium-enhanced perfusion, with pulmonary function parameters in a pilot cohort of subjects with IPF.

Six subjects with IPF were recruited. T1 mapping was performed in all subjects. Imaging sequences following inhalation of hyperpolarised 3-Helium was used to calculate estimates for ventilated volume percentage (VV\%) and coefficient of variation of ventilation $(\mathrm{CoV})$. Dynamic contrast-enhanced lung perfusion MRI was performed for pulmonary haemodynamic assessment All subjects underwent pulmonary function testing (PFTs).

VV\% strongly correlated with transfer coefficient (KCO) with $\mathrm{R}=0.955 ; \mathrm{p}=0.003$, but also FEV (forced vital capacity)/FVC ratio. $\mathrm{CoV}$ is a measure of regional ventilation heterogeneity and trended to correlation with transfer factor TLCO $(\mathrm{R}=-0.775$; $\mathrm{p}=0.108$ ). Time to peak (TTP) of the gadolinium perfusion signal showed negative correlation with FVC $(\mathrm{R}=-0.909$ with $\mathrm{p}<0.05)$ and trended to a negative correlation with TLCO $(\mathrm{R}=-0.766, \mathrm{p}=0.131)$. All $\mathrm{p}$ values two-tailed.

TTP correlation with PFT values suggests that changes in pulmonary haemodynamics may be detectable at an early stage of the disease process. VV\% and helium mapping may provide information about regional airways ventilation in IPF.
MRI based assessments could prove useful in assessing different aspects of lung structure-function for use in future research and potentially in clinical assessment of patients with IPF. Sequential imaging with concurrent PFT and echocardiography would help to further assess the applicability of evolving MRI techniques.

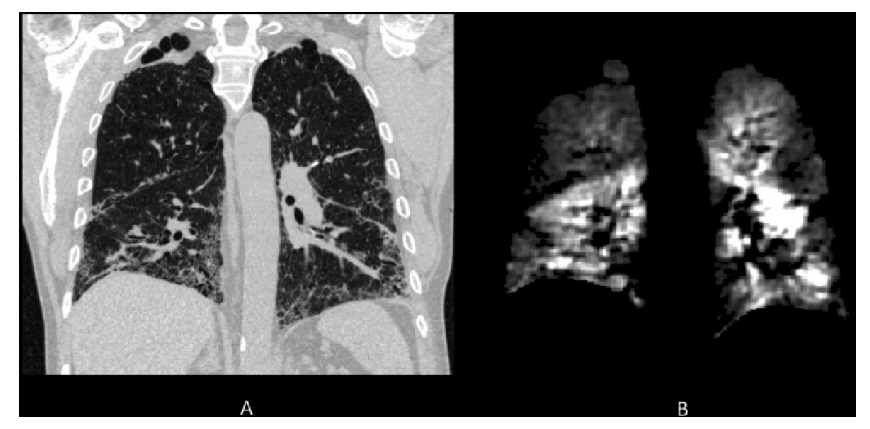

Abstract P286 Figure 1 From the same patient: coronal slice computed tomography (A); 3-Helium ventilation map (B)

\section{REFERENCE}

1 Stewart NJ, et al. Magn Reson Med. 2015;74(1):196-207

\section{P287 OFFLINE FRACTIONAL EXHALED NITRIC OXIDE AND BREATH FREQUENCY}

C Howard, V MacBean, A Lunt, A Greenough. King's College London, London, UK

\subsection{6/thoraxjnl-2015-207770.423}

Introduction/objectives Fractional exhaled nitric oxide (FeNO) is a noninvasive method of assessing airway inflammation and recommended by NICE (2014) to aid asthma diagnosis and management. Offline measurement techniques demonstrate comparable results to online and are more practical in certain clinical settings, particularly in young children who struggle with online measurements. International guidelines (ATS/ERS 2005) recommend targets for pressure and flow but not for the number of breaths per sample. Young children may take multiple breaths to complete offline reservoir filling, but whether this influences results due to contamination of the sample with ambient gas from equipment deadspace has not been assessed. Our aims were to investigate the magnitude of such effects and form a predictive equation for how increasing breath number dilutes offline measurements.

Methods A prospective observational study was undertaken recruiting 20 volunteers aged 18-42 years (13 female). FeNO was measured online (Medisoft Exp'Air 2001) and offline following exhalation into a one-litre Tedlar bag using one, five or ten breaths to complete bag filling. Airway pressure was maintained above $5 \mathrm{cmH}_{2} \mathrm{O}$ to ensure velum closure and expiratory flow at $50(+/-5) \mathrm{ml} / \mathrm{s}$.

Predicted percentages of offline FeNO relative to online were calculated by:

- 100 - ((equipment deadspace $(53 \mathrm{mls}) \times$ no of breaths)/bag volume) $* 100$

- Predicted offline values were compared to measured.

Results The median (IQR) online FeNO in parts per billion (ppb) was 24 (14-30) ppb. There was a significant reduction in offline FeNO with increasing breath number $(\mathrm{p}<0.0001)$. Median (IQR) offline FeNO of 1-breath (22 (15-32) ppb) was 